\title{
Real-time myocardium segmentation for the assessment of cardiac function variation
}

\author{
Fabian Zoehrer ${ }^{\mathrm{a}}$, Markus Huellebrand ${ }^{\mathrm{a}}$, Teodora Chitiboi ${ }^{\mathrm{b}}$, Thekla Oechtering ${ }^{\mathrm{c}}$, Malte \\ Sieren $^{\mathrm{c}}$, Jens Frahm $^{\mathrm{d}}$, Horst K. Hahn ${ }^{\mathrm{a}, \mathrm{e}}$, and Anja Hennemuth ${ }^{\mathrm{a}}$ \\ araunhofer MEVIS Institute for Medical Image Computing, Bremen, Germany \\ ${ }^{\mathrm{b}}$ NYU Langone Medical Center, New York, USA \\ ${ }^{c}$ Klinik fuer Radiologie und Nuklearmedizin UKSH, Luebeck, Germany \\ ${ }^{\mathrm{d}}$ Max-Planck-Institute for Biophysical Chemistry, Goettingen, Germany \\ ' Jacobs University, Bremen, Germany
}

\begin{abstract}
Recent developments in MRI enable the acquisition of image sequences with high spatio-temporal resolution. Cardiac motion can be captured without gating and triggering. Image size and contrast relations differ from conventional cardiac MRI cine sequences requiring new adapted analysis methods. We suggest a novel segmentation approach utilizing contrast invariant polar scanning techniques. It has been tested with 20 datasets of arrhythmia patients. The results do not differ significantly more between automatic and manual segmentations than between observers. This indicates that the presented solution could enable clinical applications of real-time MRI for the examination of arrhythmic cardiac motion in the future.
\end{abstract}

Keywords: Myocardium segmentation, real-time MRI, cardiac imaging, heart function analysis, arrhythmia

\section{INTRODUCTION}

Cardiac MR cine sequences constitute a non-invasive means for the assessment of cardiac function. The analysis of these image data can provide information about the heart muscle as well as the fraction of blood ejected from the left ventricle $(\mathrm{LV})$ per heart beat $(\mathrm{EF})$. The development of segmentation techniques enabling an quantitative extraction of heart muscle tissue as well as the blood pool of the LV has therefore been subject to image analysis research for many years. ${ }^{1,2}$ According to the guidelines, conventional cardiac cine MRI short axis sequences contain 6 to $10 \mathrm{~mm}$ tomographic cross sections representing one contraction cycle with a temporal resolution of about $50 \mathrm{~ms}^{3}$ These sequences are reconstructed from measurements during consecutive breathhold periods. New developments in real-time MR imaging already allow temporal resolutions down to $20 \mathrm{~ms}^{4}{ }^{4}$ During the same acquisition time as required for conventional acquisitions, these techniques provide ungated free-breathing direct measurements of the same tomographic slices now showing a multitude of cycles with variations caused by breathing and arrhythmia. Recent publications have shown the potential of real-time MRI to quantify the influence of arrhythmia on cardiac function. ${ }^{5,6}$ The quantitative analysis of the provided data is however a non-trivial task. The amount of data makes interactive segmentation approaches too time-consuming, and existing automatic segmentation techniques for conventional cine sequences fail to handle the contrast variations in real-time cine sequences. Only few dedicated methods exist for the segmentation of cardiac real-time MRI sequences. ${ }^{7,8}$ The purpose of the presented work is to provide and validate a fast method for the automatic segmentation of cardiac short axis real-time MRI sequences, which can serve as a basis for the quantification of cardiac function in arrhythmia patients.

Further author information: (Send correspondence to Fabian Zoehrer.)

E-mail: fabian.zoehrer@mevis.fraunhofer.de, Telephone: +49 (0)421 218-59 298

Medical Imaging 2017: Biomedical Applications in Molecular, Structural, and Functional Imaging edited by Andrzej Krol, Barjor Gimi, Proc. of SPIE Vol. 10137, 101370L · C 2017 SPIE

CCC code: $1605-7422 / 17 / \$ 18 \cdot$ doi: $10.1117 / 12.2254373$ 


\section{METHODS}

Cardiac images acquired with real-time MRI sequences exhibit some important differences compared to conventional cine MRI. The higher temporal resolution of real-time MRI changes the image contrast. Additionally real-time MRI data is acquired under free breathing, leading to potentially more motion of the organs in and through the images slices. The through-plane motion also leads to an increased appearance of saturation artefacts, which increase the gray values of the myocardium for the affected time-points. The suggested method delineates the LV myocardium in all relevant slices and time points of the real-time MRI dataset. Our automatic approach includes polar scanning transformation of the LV region followed by path detections utilizing dynamic programming techniques. These methods exhibit high computational speed while being robust to patient motion and contrast changes between slices and time points. Three main steps are explained in the following.
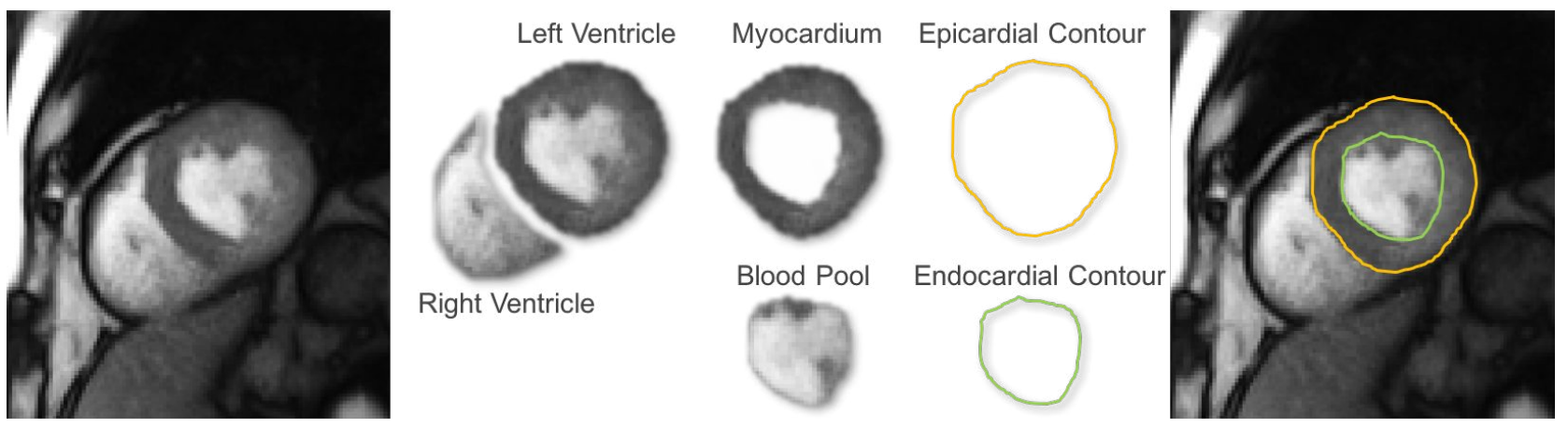

Figure 1. The goal of our work is to segment the myocardium of the left ventricle, thereby generating the epi- and endocardial contour. The above images show the separation of one short axis slice acquired with a real-time MRI technique into regions relevant to this work.

Detection of LV center point per slice: The initial preprocessing step defines a region of interest. The main goal here is to detect the center point $p_{L V \text { center }}$ of the LV in each slice, which is usually placed inside the blood pool. $p_{L V \text { center }}$ will also be used as a reference point for a polar scanning transformation in the subsequent processing steps. The blood pool appears as a roundish bright structure, so we have chosen a blob detection technique to generate candidate points. Since patients can have slight movement due to breathing and since the amount of blood in the LV is changing over time, we first project all time points per slice into a single timeframe. Multi-scale filtering is applied to each projection image (see Fig. 2). Maxima points associated to large blobs on a corresponding detail (high-pass) image are detected. Roundness and gray value strength of the blobs are calculated. Typically several candidates per slice are obtained. Candidate points of neighbor slices are grouped to chains based on their relative position on a slice. These chains of candidate points are finally analyzed based on the number of candidate points per chain, the movement of candidate positions between slices and the relative positions of the chains to each other. It has been observed that the LV position is relatively constant between slices and that there should not be any chains in the upper right area (lung). Candidate points of the selected chain are used in the following step as LV center point $p_{L V \text { center }}$ for a corresponding slice.

Detection of the epicardial contours: Epicardial contour segmentation is applied successively for all time

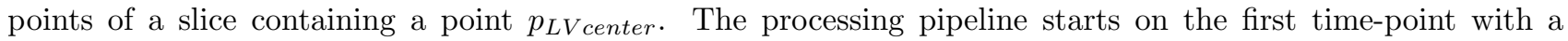
polar scanning using $p_{L V \text { center }}$ as a reference point to generate a polar image $P_{e p i}$ (see Fig. 3). We use linear resampling with a $0.2 \mathrm{~mm}$ step size to scan the circle around $p_{L V \text { center }}$ using 300 polar lines with a length of 30 to $60 \mathrm{~mm}$. Thresholding is applied to the polar image to exclude the brighter bloody and fatty parts for the following path detections. A dynamic programming technique is applied to the polar image to detect a path through the myocardium from the top to the bottom of the polar image by minimizing the variance of gray values on and near the path. The area on the left of $P_{e p i}$ is associated with the blood pool in the original image plane. Thus we use a $10 \mathrm{~mm}$ offset on the left of $P_{e p i}$ to prevent the myocardium path being placed in this area. 


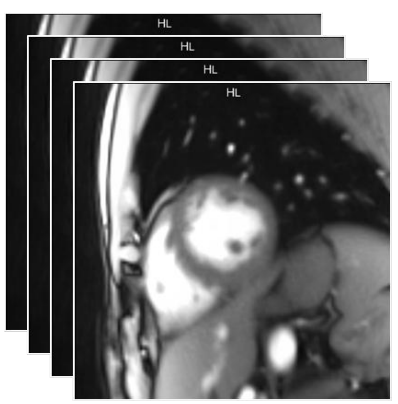

Stack of Original Image Slices

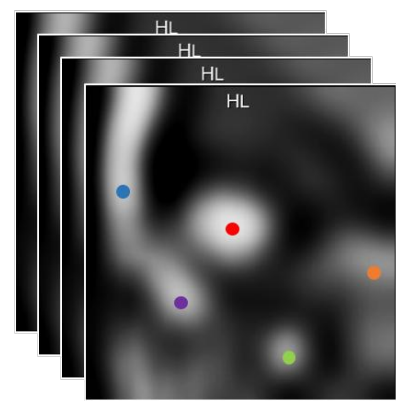

High Pass Images with Different Candidate Points

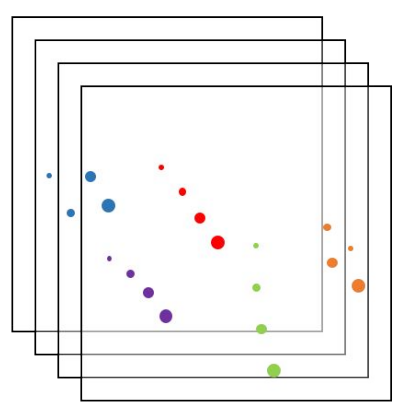

Candidate Points on Different Slices are grouped to Chains

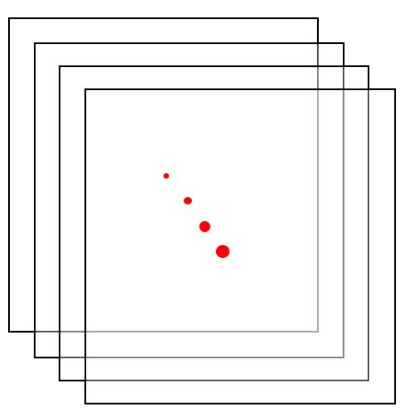

Final Chain of LV Center Points

Figure 2. LV center point detection: Each slice of a dataset is projected through time, then filtered via multi-scale filtering to produce a high pass image (second image). Multiple maxima gray value points are detected and used as candidate points for each slice. Depending on the spatial distance candidate points from neighboring slices are grouped to point chains (third image). These chains are analyzed based on relative location to each other, the movement of the candidate points through the planes and gray value strength. One chains is selected. Its points are used for the further processing of epicardial and endocardial contour segmentation.

In the next step the absolute gradient is calculated for all voxel right to the myocardium path to generate an image $G_{e p i}$. A dynamic programming processing is applied to $G_{e p i}$ to generate the path with the maxima gray value sum. A retransformation of the maxima path points from $G_{e p i}$ to the original image plane yields the final epicardial contour for a single time point. The center of gravity of this epicardial contour point is then used as an updated $p_{L V \text { center }}$ for the next time point. This way we can track patient breathing and heart beat motion.

Polar Transformation

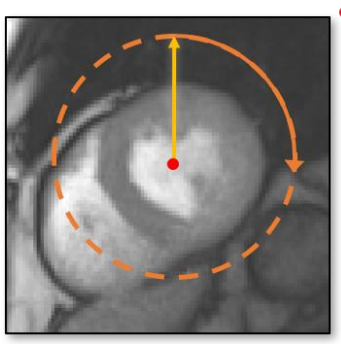

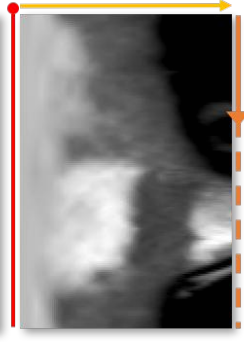

Dynamic Programming

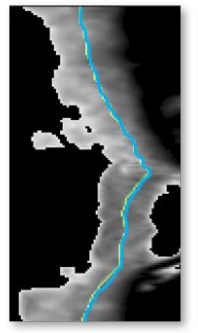

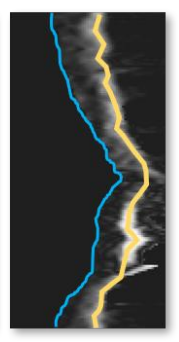

Back Transformation
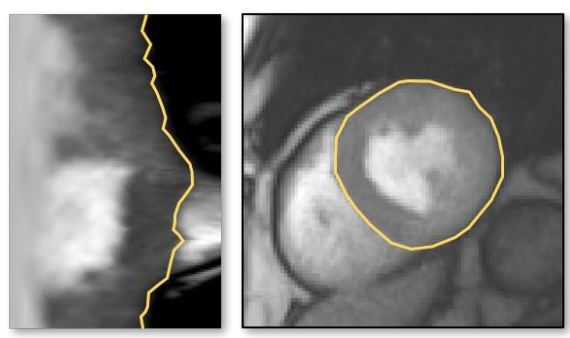

Figure 3. Overview of the epicardial contour detection. Based on the automatically detected points $p_{L V \text { center }}$ (reference point of the yellow straight polar line in the left most image) polar scanning is applied to each time point to generate a polar image $P_{e p i}$. Dynamic programming techniques are applied to the polar image $P_{\text {epi }}$ to segment a myocardium path (blue line in the third and fourth image) followed by the epicardial path (yellow line in the fourth and fifth image). Each point on the epicardial path is sampled back to the original image to yield the final epicardial contour (yellow contour in the right image) using the inverse transformation.

Detection of the endocardial contours: The epicardial contour segmentation result is used to generate a mask $M_{\text {endo }}$ of the original image comprising only the left ventricle. Another polar scanning will be applied to this mask using the center of gravity of the blood pool as a reference point. The position of the blood pool and its center point is changing through time. Therefore we calculate the mean gray value of voxels per time point and calculate the center of gravity of all voxels above this mean value. This yields the reference point $p_{\text {bloodcenter }}$. Comparable to the epicardial processing step the point $p_{\text {bloodcenter }}$ is used as reference point for polar scanning transformation yielding a polar image $P_{\text {endo }}$ (see Fig. 4). Linear resampling with a step size of $0.3 \mathrm{~mm}$ is used with 200 polar lines. The length of the lines depends on the maximal distance between pbloodcenter and the epicardial contour points. Due to the presence of papillary muscles and the myocardium structure itself the 
blood pool often appears as a fuzzy contour with concave parts. The goal is to generate a convex hull including blood pool and papillary muscles.

It is simpler and faster to generate a convex hull for a point cloud if the points are ordered based on its polar angle. Since this is always the case for a path from top to bottom in the polar image $P_{\text {endo }}$ with one voxel per row, we apply a convex hull calculation in the polar image $P_{\text {endo }}$ instead of the original image $M_{\text {endo }}$. Generating a path in $P_{\text {endo }}$ which will yield a convex hull after back transformation into the original image $M_{\text {endo }}$ is a simple

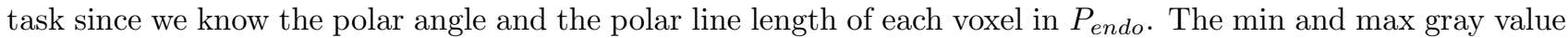
of the image mask $M_{\text {endo }}$ are used to define a set of 30 gray value bins. Each bin selects a set of points in the polar image which are used to create an associated path representing a convex hull in the original image plane. The result of this processing is a binned polar image $B_{\text {endo }}$ with a set of connected paths. Each path consist of one voxel per row with the same gray value and represents one gray value bin. These paths decrease in gray value strength from left to right of $B_{\text {endo }}$ (see third image in Fig. 4). This is equivalent to a set of convex hulls in $M_{\text {endo }}$ which decrease in gray value strength from the center point to the epicardial contour.

The next task is to select one of those paths in $B_{\text {endo }}$ as final result for the endocardial contour. A relatively simple approach has been proven to be very effective in selecting a satisfying result. We calculate the gradient in each row which yields the image $G_{\text {endo }}$. The maximum gray value per row in $G_{\text {endo }}$ is selected and the corresponding gray value in $B_{\text {endo }}$ is saved as $g(y)$. After applying this step to each row $y$ of $G_{\text {endo }}$ and $B_{\text {endo }}$, we select the lowest of the gray values $g(y)$ as $g_{\min }$. This lowest gray value $g_{\min }$ is used to select the corresponding bin and therefore one of the the paths in $B_{\text {endo }}$. This path is then finally transformed back to $M_{\text {endo }}$ and stored as the endocardial contour for the respective time point.
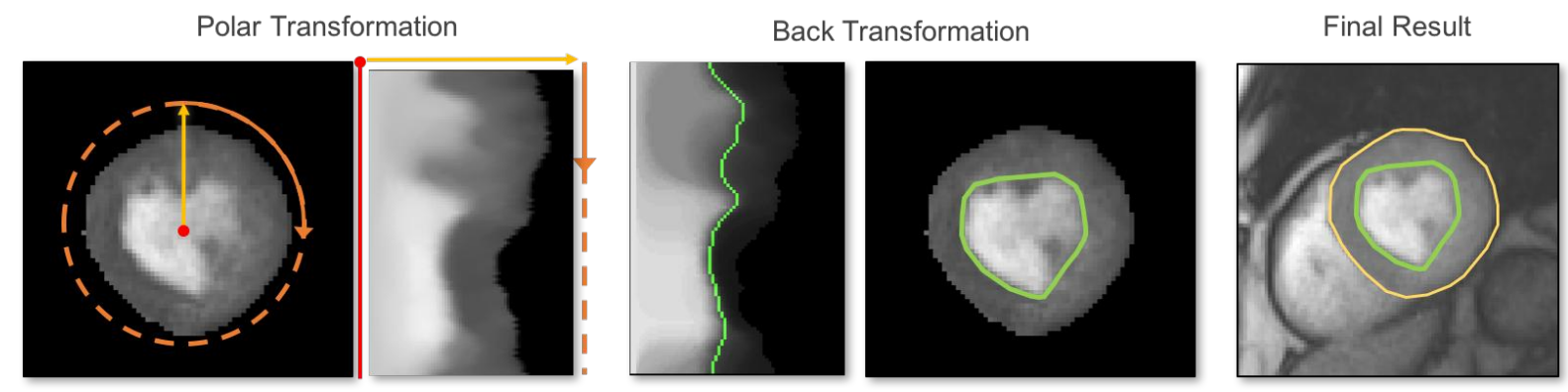

Figure 4. Overview of the endocardial contour detection. The voxel surrounded by the epicardial contour are used as

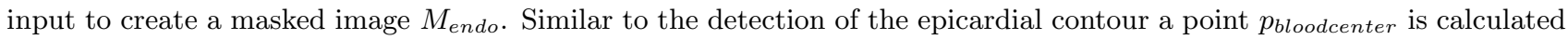
and used as reference point for polar scanning transformation to generate a polar image $P_{\text {endo }}$ (second from left). A path (green in the third image from left) in $P_{\text {endo }}$ representing a convex hull in $M_{\text {endo }}$ is calculated. Each point on this path is sampled back to the original image to generate the final endocardial contour (right image).

\section{RESULTS}

The presented method was successfully applied to 20 datasets of patients suffering from atrial fibrillation (see example screenshots in Fig. 5). Short axis cine MRI sequences covering the left ventricle over multiple heart cycles were acquired with a 3T Siemens Skyra at a resolution of $1.6 \times 1.6 \times 6 \mathrm{~mm}^{3}$ and $33 \mathrm{~ms}$ using a radial FLASH sequence. ${ }^{4}$ Reference segmentations from six experts were provided for one diastolic and one systolic timeframe per slice as well as for $10 \%$ randomly selected image frames. Each observer processed three to ten cases.

Segmentation quality of our segmentation method was assessed via Dice score, point-to-curve distance and Hausdorff distance (See table 1).

Computational Performance: The calculations have been performed on a MAC Pro (Mid 2010) with 3.33 GHz. The computational cost is around 2.5 milliseconds for a single time point or 1.5 seconds for a slice with 600 time points. For a typical dataset this makes a processing time of around 20 to 30 seconds. The processing 
Table 1. Results of evaluation: Agreement between our approach and the observers (column 1 and 2) and inter-observer agreement (column 3 and 4). Mean and standard deviation of the Dice scores, the point-to-curve distances and Hausdorff distances have been calculated for the endocardial and epicardial contours.

\begin{tabular}{|l|c|c|c|c|}
\hline & \multicolumn{2}{|c|}{ Method vs. observers } & \multicolumn{2}{c|}{ Inter-observer } \\
\hline & Endo & Epi & Endo & Epi \\
\hline Dice score & $0.88 \pm 0.03$ & $0.95 \pm 0.01$ & $0.88 \pm 0.05$ & $0.95 \pm 0.01$ \\
\hline Point-to-curve distance $(\mathrm{mm})$ & $2.01 \pm 0.33$ & $1.33 \pm 0.22$ & $1.97 \pm 0.49$ & $1.20 \pm 0.15$ \\
\hline Hausdorff distance $(\mathrm{mm})$ & $5.21 \pm 0.78$ & $4.29 \pm 0.58$ & $4.49 \pm 1.12$ & $3.39 \pm 0.31$ \\
\hline
\end{tabular}

of a dataset is faster than its acquisition, since the temporal distance between the acquisition of two time points is 33 milliseconds. If necessary the speed could be improved further by using parallelization.
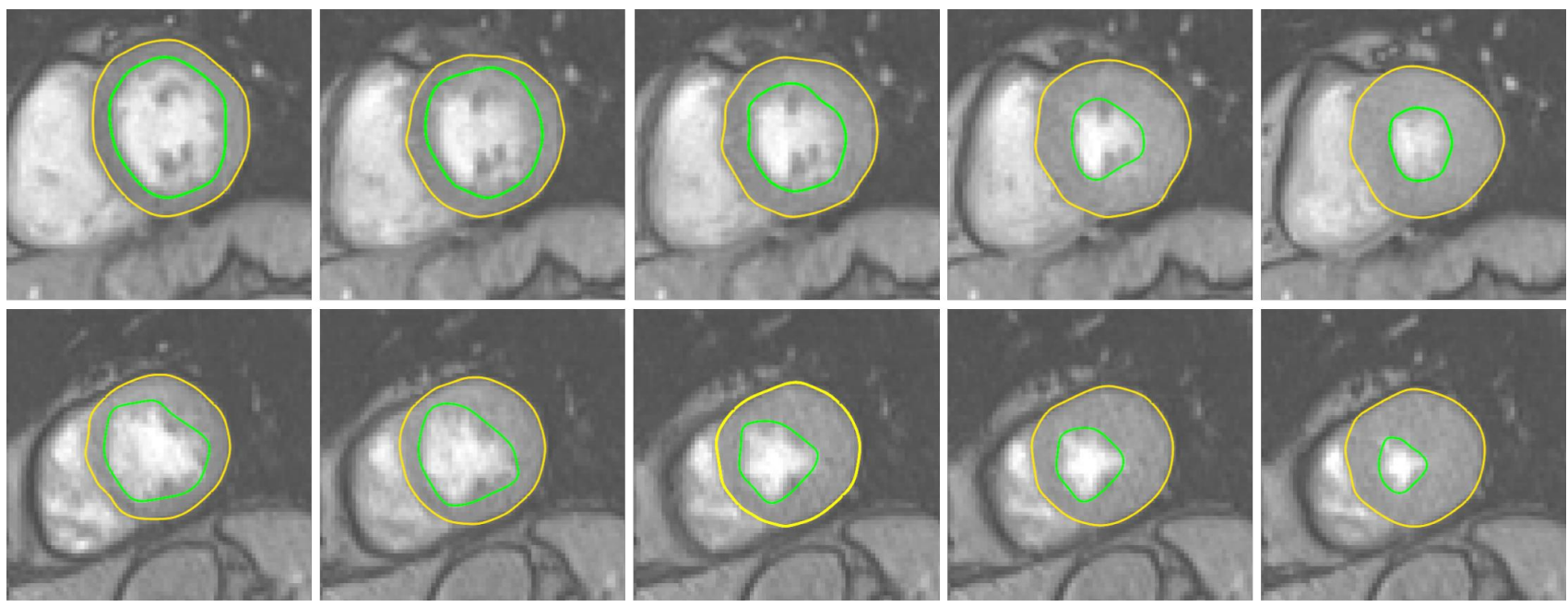

Figure 5. These images show the results of the epicardial (outer, orange) and endocardial (inner, green) contour detection for five time points on two different slices (top and bottom). All results are computed automatically with a processing speed of 2.5 milliseconds per time point.

Integration in the clinical environment: The segmentation algorithm has been integrated into our CaFur application (Fig. 6). CaFur is a client-server application for the quantitative analysis of multi-cycle real-time MRI data of blood flow and cardiac function. This approach combines an automatic image processing with an interactive exploration of the additional information provided by the real-time MRI methods such as beat-tobeat variability of clinical parameters. To assess the parameters such as end-systolic (ESV) and end-diastolic volume (EDV), stroke volume (SV), ejection fraction (EF) and myocardial mass image sequence is separated into cardiac cycles based on the automatic segmentation. The solution enables the measurement of the influence of arrhythmia on cardiac function as well as a comparison of cardiac function with and without physical stress.

\section{CONTRIBUTION}

State-of-the-art imaging technology enables the acquisition of cardiac real-time MRI data for the assessment of cardiac function during free breathing as well as arrhythmia. There are however no commercial tools available for the analysis of these data. Published approaches from researchers present methods, which require high computational and interaction effort. ${ }^{7,8}$ The presented segmentation approach constitutes a solution for fast processing of short axis real-time MRI cine sequences. The adaptation of the polar scanning approach enables 


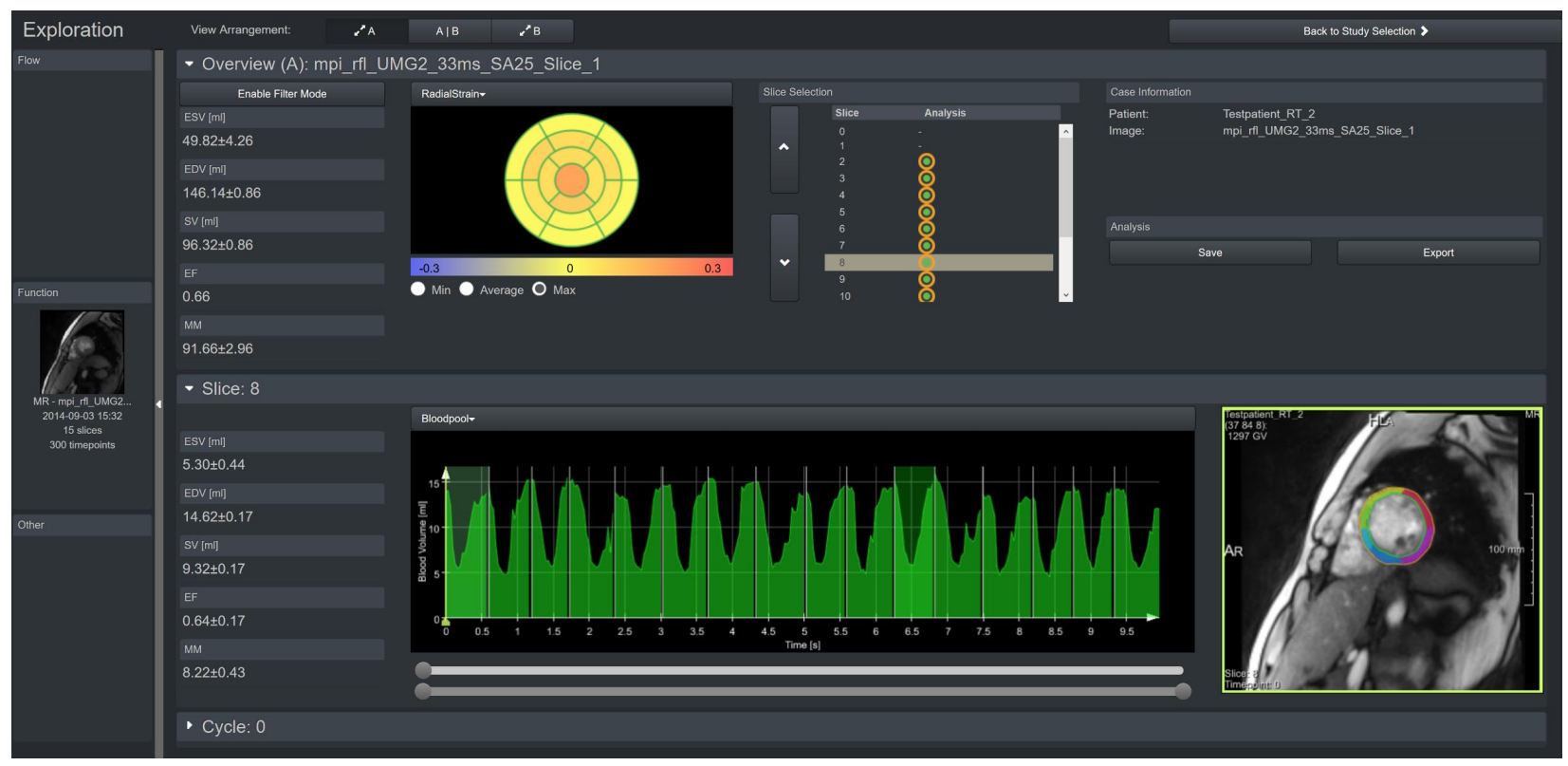

Figure 6. Example screenshot of the CaFur application which uses the myocardium segmentation presented in this work to calculate and present heart function parameters. The central bottom image shows the blood pool volume curve for a selected slice. For more information about CaFur: https://www.mevis.fraunhofer.de/en/solutionpages/ solutions-for-cardiac-imaging.html.

processing of cine MRI slice sequences within seconds and could be integrated in an automatic preprocessing pipeline. The presented results show the applicability to patient data acquired under free breathing. Thus, the presented segmentation approach could enable the usage of real-time MR imaging for the quantification of cardiac function and its variation in a clinical setting.

\section{CONCLUSIONS}

We have presented a fast automatic segmentation method for cardiac real-time MRI short axis sequences. The suggested approach performs an initialization via a center point detection for the LV. The subsequent polar scanning steps find the myocardial contours. The presented results show a good agreement with the reference annotations by expert observers. The systematic differences between the segmentations by observers from different institution suggest that it might be helpful to enable parameterizations according to the segmentation paradigms of the users. In future work we aim to integrate our method with interactive correction and quantification methods in order to enable the application in clinical research.

\section{ACKNOWLEDGMENTS}

The authors would like to thank Prof. Christina Unterberg-Buchwald for providing the real-time MRI data analyzed in work presented in this paper.

\section{REFERENCES}

[1] Petitjean, C. and Dacher, J.-N., "A review of segmentation methods in short axis cardiac MR images," Med. Image Anal. 15(2), 169-184 (2011).

[2] Peng, P. et al., "A review of heart chamber segmentation for structural and functional analysis using cardiac magnetic resonance imaging.," MAGMA 29(2), 155-195 (2016).

[3] ACCF et al., "Accf/acr/aha/nasci/scmr 2010 expert consensus document on cardiovascular magnetic resonance: a report of the american college of cardiology foundation task force on expert consensus documents.," J Am Coll Cardiol 55(23), 2614-2662 (2010). 
[4] Zhang, S. et al., "Real-time magnetic resonance imaging of cardiac function and flow-recent progress.," Quantitative imaging in medicine and surgery 4(5), 313-29 (2014).

[5] Unterberg-Buchwald, C. et al., "Real time cardiac MRI and its clinical usefulness in arrhythmias and wall motion abnormalities," Journal of Cardiovascular Magnetic Resonance 16 (2014).

[6] Contijoch, F. et al., "Quantification of left ventricular function with premature ventricular complexes reveals variable hemodynamics.," Circ Arrhythm Electrophysiol 9(4), e003520 (2016).

[7] Chitiboi, T. et al., "Context-based segmentation and analysis of multi-cycle real-time cardiac MRI," IEEE ISBI , 943-946 (2014).

[8] Contijoch, F. et al., "User-initialized active contour segmentation and golden-angle real-time cardiovascular magnetic resonance enable accurate assessment of LV function in patients with sinus rhythm and arrhythmias.," Journal of Cardiovascular Magnetic Resonance 17, 37 (2015). 